\title{
Technical Feasibility of a Robotic-Assisted Ventral Hernia Repair
}

\author{
Guido Beldi
}

Published online: 29 December 2011

(C) Société Internationale de Chirurgie 2011

Incisional hernia is one of the most frequent complications after abdominal surgery. Its incidence is typically underestimated and may range up to $26 \%$ [1]. Thus, novel and potent strategies to prevent and treat incisional hernia are required. Implantation of a prosthetic mesh has become the "gold standard" of open and laparoscopic incisional hernia repair. Laparoscopic mesh implantation is associated with the typical advances of laparoscopy, such as a lower rate of surgical site infections and reduced hospital stay [2, 3]. However, hernia recurrence has not been reduced significantly by the laparoscopic approach and long-term pain remains a therapeutic challenge in a subset of patients $[4,5]$.

In this issue of the Journal, Allison and colleagues [6] explored a novel therapeutic strategy. By using the Da Vinci Robot, the authors took advantage of facilitated intracorporeal suturing. Thus, adaptation of the hernial orifice, which may be demanding during laparoscopic surgery, was mastered using the robot. Because of the technical difficulties, this operative step is not performed during many laparoscopic incisional hernia repairs. Closing the hernial orifice may play an important role, because it reduces a gap in the abdominal wall, which in turn may result in migration of the mesh by being pushed into this gap by elevated intraabdominal pressure. Here, robotic surgery potentially facilitates this step, which might be of importance to a lower recurrence rate.

The authors also used the robot for mesh fixation using running sutures and thereby avoiding the insertion of transfascial sutures or metal tacks. Either of these fixation

G. Beldi $(\bowtie)$

Department of Visceral Surgery and Medicine,

Bern University Hospital, Bern, Switzerland

e-mail: Guido.Beldi@insel.ch devices, or combinations, is nowadays required for effective mesh fixations but is associated with specific risks [5].

Robotic surgery, however, is time-consuming and associated with increased costs. Until now, no significant advances for general abdominal surgery have been shown and the robot failed to be introduced widely. But why? In our own experience, we performed robotic surgery in 21 robotassisted laparoscopic groin hernia repair (TAPP). The procedure was technically feasible and helped to improve our skills in the use of the robot. However, the advantages of laparoscopic repair were not improved by the robot. Neither safety nor morbidity was superior compared with retrospective series. In particular, costs were increased by $34 \%$ by using the robot.

Similarly, in the present article [6], the authors described the feasibility and safety of robot-assisted incisional hernia repair. Some of the achievements have to be regarded with caution, because the authors used the robot for rather small hernias, which are typically not associated with elevated risk of recurrence and pain. Thus, it will be a long way to show any improvement of robot-assisted incisional hernia repair compared with conventional laparoscopic procedures. In particular, the field of laparoscopic incisional hernia repair is still moving forward and critical steps, such as fascial closure, the perfect type of mesh, or the perfect fixation method, are currently being investigated. Thus, there is currently no "gold standard" for the comparison with robotic repair, including open repair. The authors, or other investigators, will have to show an improvement by robotic incisional hernia repair, e.g., lower recurrence rate or less pain, to justify such expensive procedures. Furthermore, current robots have known disadvantages: notably the visualization of large areas and working in different abdominal regions is still difficult-both of which are required for laparoscopic ventral hernia repair. Thus, robots need to be improved 
significantly by introducing novel technologies to enable the surgeon to benefit from its advantages and potentially allow its widespread use for incisional hernia repair.

\section{References}

1. Sorensen LT, Hemmingsen UB, Kirkeby LT et al (2005) Smoking is a risk factor for incisional hernia. Arch Surg 140:119-123

2. Sauerland S, Walgenbach M, Habermalz B et al (2011) Laparoscopic versus open surgical techniques for ventral or incisional hernia repair. Cochrane Database Syst Rev CD007781
3. Forbes SS, Eskicioglu C, McLeod RS et al (2009) Meta-analysis of randomized controlled trials comparing open and laparoscopic ventral and incisional hernia repair with mesh. Br J Surg 96: 851-858

4. Sajid MS, Bokhari SA, Mallick AS et al (2009) Laparoscopic versus open repair of incisional/ventral hernia: a meta-analysis. Am J Surg 197:64-72

5. Beldi G, Wagner M, Bruegger LE et al (2011) Mesh shrinkage and pain in laparoscopic ventral hernia repair: a randomized clinical trial comparing suture versus tack mesh fixation. Surg Endosc 25:749-755

6. Allison et al (2011) Technical feasibility of a robotic assisted ventral hernia repair. World J Surg (in press). doi:10.1007/s00268011-1389-8 